\title{
LA NATURALEZA DE LA VIDA REFLEJADA EN EL DISEÑO
}

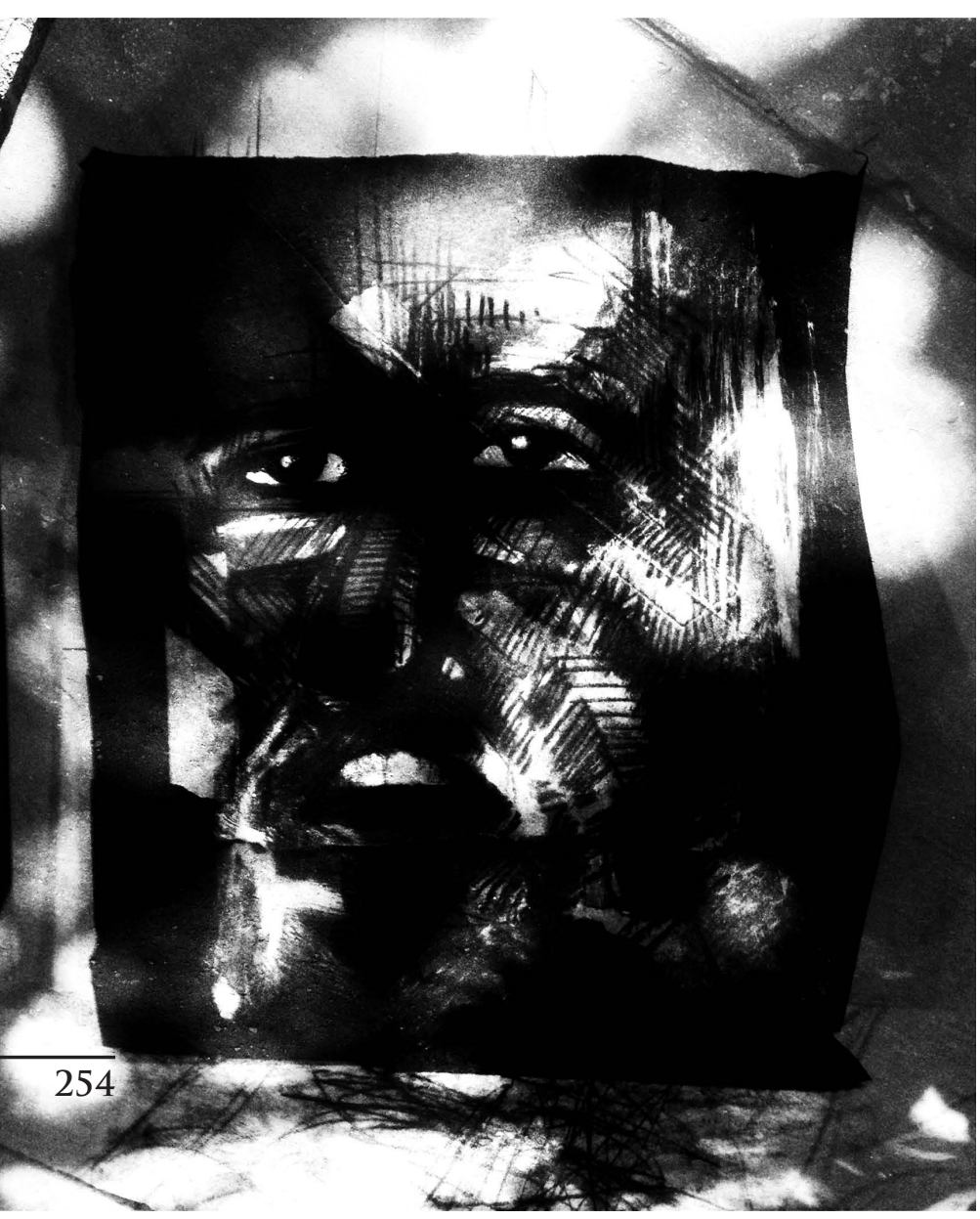

THE NATURE OF LIFE REFLECTED ON DESIGN

\author{
A NATUREZA DA VIDA \\ REFLETIDA NO DESIGN
}

Por:

\section{Diomar Elena Calderón Riaño}

Profesora

Universidad San Buenventura, Medellín (Colombia)

diomar.calderon@usbmedellin.onmicrosoft.com

Ilustración: Andrés Reina Gutiérrez

Resumen: Algunos principios de la naturaleza de la vida aplicados en "Diseño para la Innovación Social”; un curso que acerca a escenarios reales, dando la oportunidad de afinar los sentidos en la lectura de situaciones, estimulando la comunicación y el pensamiento. Esta recopilación crea un registro como bitácora de vuelo, para no dejar en el olvido aquellos recorridos hechos, los hallazgos ya encontrados; dando apertura a lo que está por descubrirse. Actualmente el ejercicio académico participa en alianza con la Red Académica de Diseño y la Agencia Nacional para la Superación de la Pobreza Extrema; en una red articulada para llevar el aula a escenarios reales que inspiran procesos de diseño.

Palabras clave: redes, pensamiento sistémico, diseño social 


\begin{abstract}
Some principles of the nature of life applied to "The Design for Social Innovation", a course of action that bring closer real scenarios, giving the opportunity to enhance the senses during the reading of situations, promoting communication and thought. This compilation creates a register, like a flight log, in order not to forget those paths, findings; opening the door to what is yet to be discover. Nowadays, this academic attempt takes part in the alliance between the Red Académica de Diseño and the Agencia para la Superación de la Pobreza Extrema; in a network intended to take the classroom to real scenarios that inspire design processes.
\end{abstract}

Keywords: Webs, systematic thought, social design.

Resumo: Alguns princípios da natureza da vida aplicados em Design para a inovação social, um curso que aproxima a cenários reais, dando a oportunidade de afinar os sentidos na leitura de situações, estimulando a comunicação e o pensamento. Esta recopilação cria um registro, como um registo de voo, para não deixar no olvido aquelas viagens feitas, descobrimentos; abrindo a porta a novos descobrimentos a ser feitos. Atualmente o exercício acadêmico faz parte da aliança da Red Académica de Diseño e a Agencia Nacional para la Superación de la Pobreza Extrema; a qual é uma rede articulada para levar a aula a cenários reais que inspiram processos de design.

Palavras-chave: Redes, pensamento sistêmico, design social.

La inmediatez evidente por la respuesta en un contexto donde todo parece en emergencia constante, hace necesario que la academia actúe como regulador de esa velocidad para pensarse el sentido de la disciplina del Diseño con relación a los cambios de la sociedad, explorar nuevas ideas y modos de pensarlo, buscar descripciones extensivas y holísticas de las situaciones para establecer un enfoque sistémico del espectro de los fenómenos y experimentar el impacto de aprender a ver el todo con el valor y sentido de sus relaciones.

¿Qué implica ser vivo? ¿Cuáles son las características de la vida? ¿Y de qué forma esta condición natural se refleja en las actividades, especialmente aquellas vinculadas al contexto educativo? la primera condición, y tal vez obvia, que favorece los procesos de diseño en un aula, es que somos seres vivos; y la segunda es que existe una intención común relacionada con la pasión compartida por el Diseño; ambas suficientemente potentes como para generar procesos relevantes en el aula. 


\section{Primera condición: acerca de las implicaciones de ser vivo}

\section{La red}

El ser humano es un sistema vivo que tiene en su estructura una serie de componentes que se relacionan entre sí para el cumplimiento de funciones vitales, y corresponden a los objetivos comunes del sistema. La teoría de los sistemas considera el estudio de la substancia o estructura que se puede medir y pesar, información de tipo cuantificable, y por otro lado el estudio de la forma o patrón como elemento que no puede ser medido ni pesado; para su comprensión se requiere la cartografía de la configuración de sus relaciones ordenadas (Capra , 1996). De esta forma, para entender lo sistémico se hace necesaria la comprensión de la vida a partir de la comprensión del patrón. Existe entonces algo inmaterial e irreducible: el patrón de organización.

La propiedad más importante del patrón de organización, es que se trata de un patrón en forma de red. Dondequiera que encontremos sistemas vivos, organismos, partes de organismos o comunidades de organismos-, podremos observar que sus componentes están dispuestos en forma de red. (Capra , 1996, p. 100)

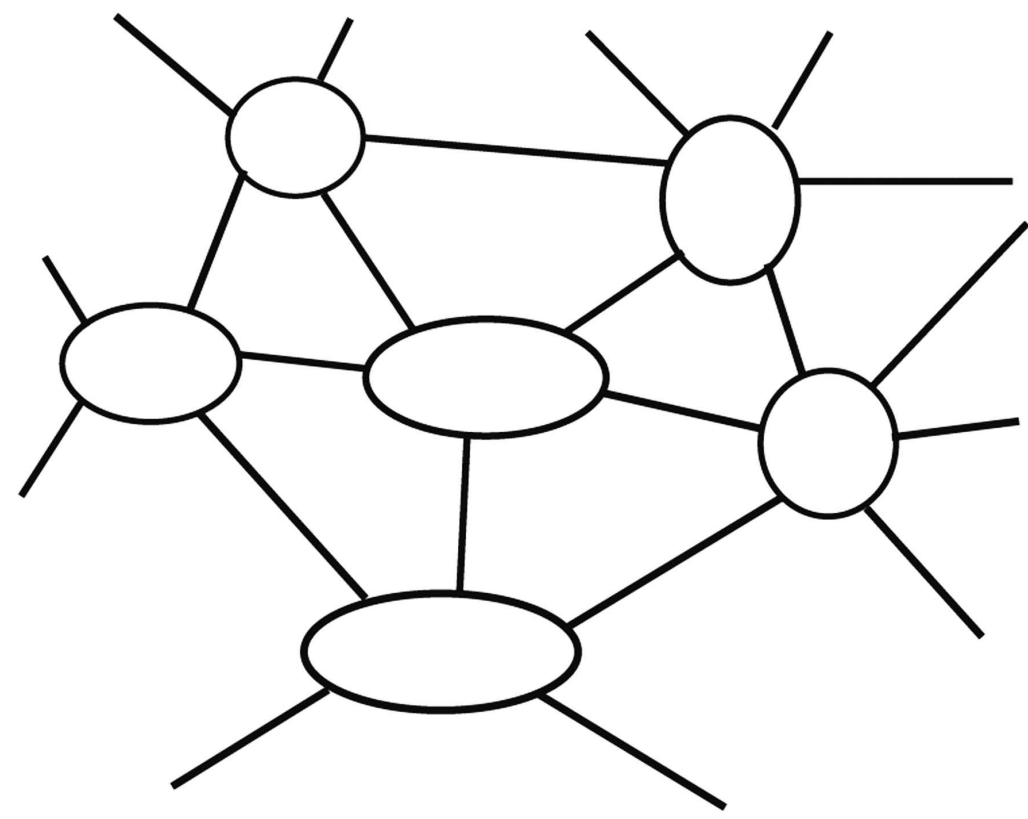

Figura 1. Esquema del patrón de red

El patrón contiene la organización de las relaciones. Por ejemplo, si un sistema vivo es diseccionado, sus componentes vistos individualmente siguen ahí, pero la relación establecida con los otros componentes se ve deteriorada, es decir, su patrón se destruye y en consecuencia el organismo muere; las propiedades sistémicas se ven afectadas cuando el sistema se disecciona en elementos aislados (Capra , 1996). 


\section{Auto-organización que evoluciona a Autopoiesis}

A partir del reconocimiento de la red como patrón general de la vida se origina el concepto de auto-organización, el cual es definido posteriormente por los autores Maturana y Várela como autopoiesis, entendida como la organización común a todos los sistemas vivos.

La auto-organización asigna a los sistemas las siguientes características: la primera y más importante es que se trata de un patrón en forma de red, esto significa que ya sean partes, comunidades o simplemente organismos van a estar dispuestos en forma de red, es decir "si vemos vida, vemos redes". Los sistemas vivos son sistemas abiertos que se accionan alejados del equilibrio, estado que posibilita la emergencia de nuevas estructuras y nuevos modos de comportamiento; esto hace que se requiera una entrada constante de materia y energía para que pueda darse la auto-organización, lo cual corresponde a la segunda característica común a estos modelos. Y, por último, los modelos de auto-organización tienen como característica común a todos los modelos, la interconectividad no-lineal de los componentes del sistema, lo cual se refleja físicamente en bucles de retroalimentación (Capra, 1996).

Autopoiesis como concepto se separa en Auto que se refiere a la autonomía de los sistemas auto- organizadores, significa sí mismo, y Poiesis, que tiene la misma raíz griega que poesía, significa creación. De esta forma Autopoiesis significa «creación de sí mismo».

La autopoiesis se trata de una red de procesos de producción, en la que la función de cada componente es participar en la producción o transformación de otros componentes de la red. De este modo toda la red se «hace a sí misma» continuamente. Es producida por sus componentes y, a su vez, los produce. «En un sistema vivo», explican los autores, «el producto de su operación es su propia organización.» (Capra, 1996, p. 116).

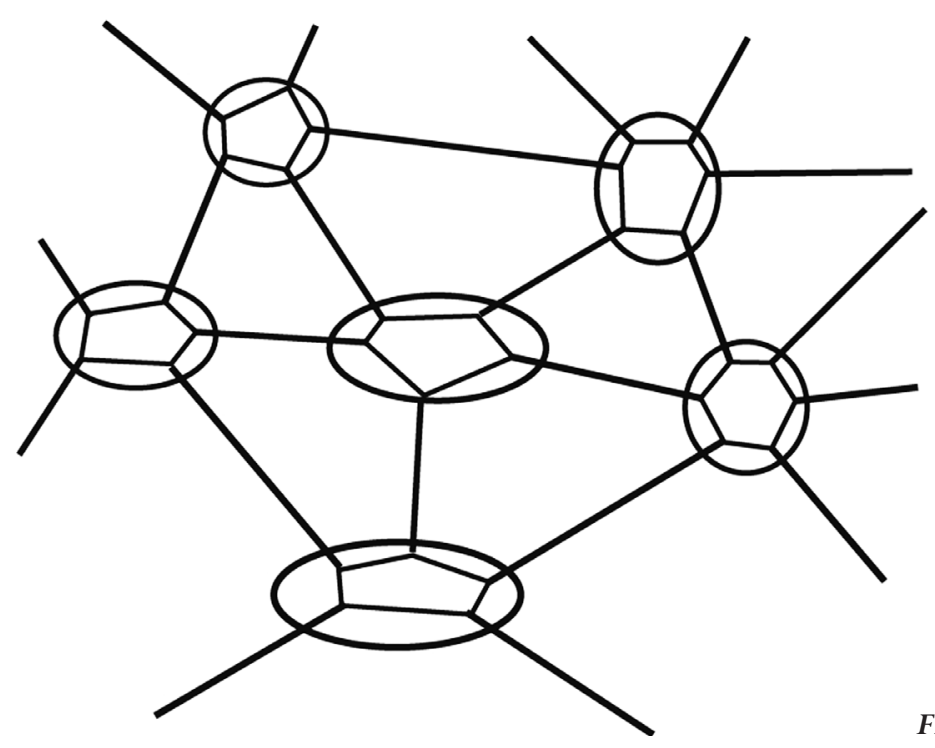

Figura 2. Producción de sí mismo, autopoiesis 


\section{Autorregulación relacionada con retroalimentación}

Los elementos en un sistema vivo son elementos conectados causalmente, el bucle indica una disposición circular, en donde la causa inicial genera efectos en los puntos sucesivos; de esta forma cada punto tiene su efecto en el siguiente, retroalimentando el punto de partida donde inició: "Los bucles de retroalimentación son patrones abstractos de relaciones inmanentes en actividades de organismos vivos, implica el retorno de la información a su punto de origen” (Capra, 1996, p. 82).

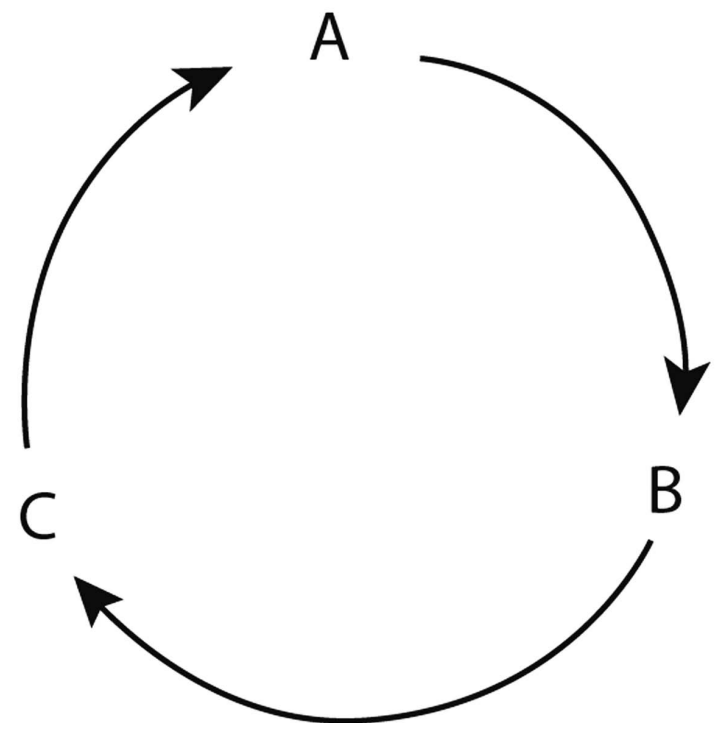

Figura 3. Causalidad circular en un bucle de retroalimentación

La entrada generada en el primer eslabón (input) se ve influenciada por el último eslabón (output), lo cual genera procesos auto-reguladores característicos del sistema vivo; allí el punto de partida se ve modificado a lo largo de cada punto del recorrido. Emerge evidentemente una retroalimentación entendida como el retorno de la información al punto inicial por medio de un proceso o actividad, la cual actúa en los organismos vivos como facilitadora del estado de equilibrio dinámico (Capra, 1996).

\section{Vida y cognición}

El entendimiento de un sistema vivo requiere del estudio del patrón (forma, orden, cualidad) y el de la estructura (substancia, materia, cantidad); pero aquí los componentes cambian continuamente, hay un flujo de materia y energía a través del organismo; esto genera en la comprensión de la estructura viva el inseparable entendimiento de los procesos metabólicos y relativos al desarrollo. Esta propiedad de los sistemas vivos sugiere el proceso como tercer criterio para una completa descripción de la naturaleza de la vida. El proceso vital es la actividad que se ocupa de la continua corporeización del patrón de organización del sistema. De esta forma el criterio de proceso constituye el vínculo entre patrón y estructura en un sistema vivo. (Capra, 1996). 
Los tres criterios son totalmente interdependientes. El patrón de organización sólo puede ser reconocido si está corporeizado en una estructura física, siendo éste un proceso continuo en los organismos vivos. Así pues, estructura y proceso están inextricablemente unidos. (Capra, 1996, p. 173)

De acuerdo con la teoría de los sistemas vivos, la mente es el proceso mismo de la vida. En otras palabras, la actividad organizadora de los sistemas vivos es una actividad mental. Las interacciones de un organismo vivo con su entorno son interacciones cognitivas, mentales, así, vida y cognición quedan inseparablemente vinculadas. El proceso mental es inherente en la materia a todos los niveles de vida, entonces la percepción no puede ser contemplada como la representación de una realidad externa, sino que debe ser entendida como la creación continua de nuevas relaciones en el interior de la red neuronal. El proceso de vivir es un proceso de cognición, por lo tanto cualquier aprendizaje no es temporal es un asunto que trasciende la vida (Capra, 1996).

La percepción del mundo viviente como una red de relaciones ha convertido el pensamiento en términos de redes en otra de las características fundamentales del pensamiento sistémico es decir en términos de conectividad, relaciones y contexto. Las propiedades esenciales de sistema viviente emergen de las interacciones y relaciones entre las partes las cuales no están aisladas y la naturaleza del conjunto es siempre distinta de la mera suma de sus partes. El pensamiento sistémico es contextual, es decir encuadra este algo dentro del contexto de un todo superior. (Capra, 1996, p. 48)

\section{Segunda condición: acerca de la pasión del diseño}

Según John Dewey "el desarrollo humano sano depende de las capacidades individuales para integrar las experiencias sucesivas con otros” (Margolin, 2002). La creación de una realidad social está influenciada por las acciones de los individuos de una sociedad y del diseño, entendido como un elemento que pertenece a la comunidad, cuya responsabilidad es mejorar la calidad de vida de las personas.

El diseño desde su enfoque social tiene un número de ejes o valores fundamentales y se preocupa por las necesidades reales de las personas, hábitos y cultura creados en una comunidad, que conforman la red formada por una red de relaciones entre las personas y las acciones con impacto a nivel social y ambiental.

La responsabilidad social y moral, como valor importante "se basa en las decisiones que tomamos todos los días" en los productos que creamos, en las interacciones que tenemos con el medio ambiente y el estilo de vida que llevamos. Así el diseño social tiene la responsabilidad de favorecer la realidad social, y a su vez, la sociedad tiene la responsabilidad de los cambios en el entorno en que vive. 
Según Thackara, el diseño comprometido con el contexto social del consumo y de las comunidades, promueve la colaboración y la innovación. Este diseño colaborativo produce no sólo los objetos, es responsable de la generación de ideas, conocimientos, procesos y relaciones. Para Jegou y Manzini, la sociedad deja de ser vista como un hecho aislado y se convierte en un diseño "red de redes", en la que se interconectan los procesos y el diseñador se concibe como parte de la comunidad, y, como actor en los procesos de ayuda en el desarrollo creativo de la comunidad que está trabajando (Co-Design).

La innovación como un proceso social implica interacciones entre los individuos y las comunidades de la sociedad, allí el diseño se percibe como un medio para impulsar a través de la creación de un entorno que ofrece la oportunidad de compartir ideas y aprender de las experiencias de los demás; este proceso también se conoce como la innovación social. La participación activa de los individuos y la comunidad genera mayores oportunidades para proponer diseños compatibles con el contexto, facilitando el pensamiento colaborativo, integrando personas, mejorando comunicación, favoreciendo el fortalecimiento de la red y, por ende, al intercambio de conocimientos.

Como resultado de todos los valores, el diseño social promueve nuevas soluciones sostenibles en los escenarios de la vida cotidiana, el rechazo de los excesos y de bienestar que ofrece nuevas ideas en un entorno compuesto por las organizaciones en red, con un sentido de horizontalidad donde todos los elementos son igualmente importantes. Organizaciones descentralizadas abiertas para sistemas más grandes, que favorecen el trabajo y la responsabilidad compartida entre todas las personas en la sociedad.

Algunos principios de los sistemas vivos son aplicados en "Diseño para la Innovación Social", un curso que acerca a escenarios reales para afinar los sentidos en la lectura de situaciones, estimular la comunicación y el pensamiento. Esta recopilación crea un registro para no dejar en el olvido aquellos recorridos hechos, dando apertura a lo que está por descubrirse en las dinámicas propuestas en el aula.

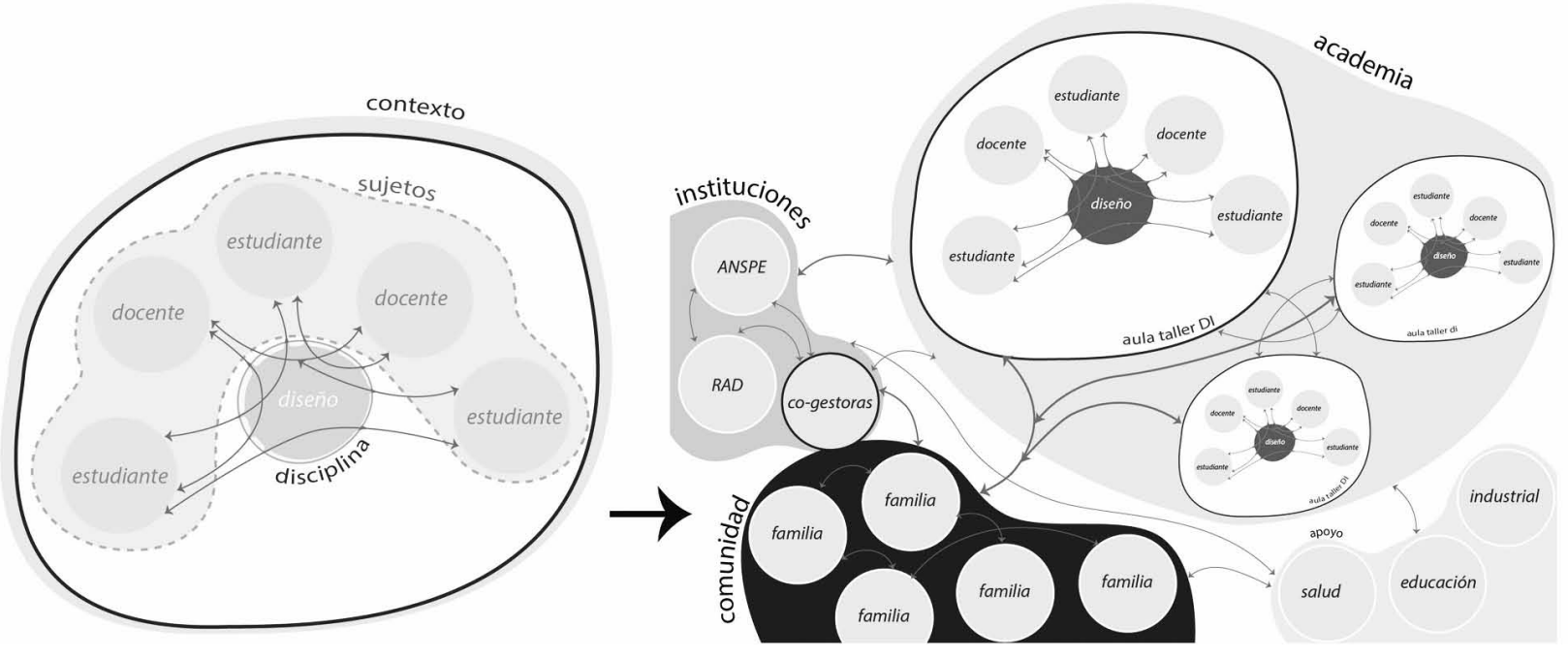

Figura 4. La red en el aula 
En la figura 4 se evidencian los sujetos participes, la relaciones, el contexto que representa la membrana que determina los recursos disponibles y limita la acción de este sistema. Aquí se vincula la segunda condición donde el Diseño actúa como el propósito común capaz de generar relaciones en el sistema, las cuales son representadas con líneas fluidas entre los sujetos involucrados, dando mayor fuerza al conocimiento y las experiencias dadas en los procesos en el aula.

Si se habla Diseño en Comunidad, el aula no se puede limitar al aula física, sino que debe trascender y generar otros nodos de red, grandes grupos localizados que se interrelacionan, como las Academias que constituyen una serie de universidades en el país que vinculan los ejercicios del aula con el desarrollo de propuestas para la alianza Taller RAD-ANSPE. Esta alianza se localiza en el grupo de las Instituciones, que tienen como función planear y gestionar una serie de acciones que son implementadas a nivel nacional y regional, hasta llegar a una escala local a través de figuras como las cogestoras; éstas se articulan como sujetos fundamentales por su función de puentes entre las entidades y la Comunidad, y permiten consolidar las funciones en la red para llevar a un plano operativo las estrategias planteadas para incrementar los niveles de calidad de vida de las familias beneficiarias de los programas.

Las redes de comunicación pueden generar retroalimentación; estudiantes, docentes y comunidad partícipes se involucran para aprender de sus experiencias, donde el impacto llega a todos y vuelve al origen. La autorregulación emerge como el concepto central de la visión sistémica de la vida y al igual que los conceptos de retroalimentación y organización que están íntimamente ligados a las redes. (Capra, 1996, p. 76).

\section{Momentos claves en el Proceso de Diseño}

El Diseño actúa como facilitador entre los sujetos involucrados, representa entonces el concepto del interés común para las partes en diferentes niveles. El Pensamiento de Diseño es un asunto complejo, no existen recetas únicas para llegar a un buen diseño, pero si es importante establecer momentos claves en el proceso creativo cuyos alcances, tratándose de un curso, están directamente relacionados con las variables de tiempo y espacio.

El proceso involucra la subjetividad del diseñador, vincula la forma de ver e interpretar el mundo en el ejercicio proyectual. La incerteza en el proceso creativo existe, pero durante el ejercicio los objetivos y requerimientos se definen y actúan como pautas en el proceso de diseño. Y por último y no menos importante hay "magia” porque la idea viaja, se exterioriza para llegar a ser transformada en un elemento activo en la construcción de sociedad.

El proceso contempla los siguientes momentos claves en el desarrollo del curso: 
Momento 1: Descubrir para encontrar sentido

"La realidad de la que podemos hablar nunca es la realidad a priori, sino una realidad conocida, la cual le damos forma"(Heisenberg)

Este ejercicio de diseño pretende en esencia evidenciar el rol del diseñador industrial en contextos sociales que actúa en situaciones y problemáticas vinculadas a la comunidad, partiendo de la exploración, el conocimiento y la interpretación de las dinámicas que suceden en el contexto. Este primer contacto genera en el estudiante el desarrollo de habilidades para la observación, la comunicación y la empatía para percibir al otro no como objeto de estudio sino como sujeto activo en la configuración del proyecto. La percepción hace indispensable mirar al observador con referencia a lo observado, yendo un poco más allá de Heisenberg "acerca del efecto de cualquier observación sobre el objeto, en función de que siempre la distinción que se traza de un universo involucra a un perceptor que la ejecuta". (Ceberio \& Watzlawick, 1998, p. 66)

A medida que pasa el tiempo, el ser humano construye su forma de ver el mundo y aprender en él; es un proceso continuo, y conocer estas formas propias es fundamental para iniciar cualquier proceso en el aula, ya que esto garantiza un proceso basado en los ritmos de cada participante en el proceso de aprendizaje. "El punto de partida es la conciencia del conocimiento del mundo, y de cómo me relaciono con él y con los demás que lo habitan”. (Ceberio \& Watzlawick, 1998, p.28)

El ser humano en su desarrollo evolutivo, como parte del proceso de adaptación al medio ambiente, intenta edificar una estructura mental que le permita ordenar esa tendencia a la entropía de su experiencia y, a través de este proceso, irá estableciendo experiencias repetibles y relaciones más o menos confiables, construyendo así un mundo el cual llama realidad. (Ceberio \& Watzlawick, 1998, p.74).

El planteamiento acerca del sentido de interacción entre el sujeto y el medio como forma para la construcción individual de la realidad, evidencia la relación entre la adquisición del conocimiento y el modelo de la realidad, que definido desde un punto de vista evolutivo hace referencia a que un organismo nunca es capaz de reconocer y describir la realidad; simplemente construye un modelo que le permite avecinarse a ella. Así la comunicación hace que dos o más sujetos que se relacionan construyan un mundo colectivamente. De esta relación emerge la vida social, siendo el lenguaje una de sus consecuencias (Ceberio \& Watzlawick, 1998).

Todos los seres humanos estamos limitados por nuestro aparato perceptivo y tanto nuestra experiencia como los objetos de la misma son el resultado de nuestra forma individual de experiencia, o sea, están estructurados y determinados por nuestras categorías de espacio y de tiempo, y nunca es capaz de captar la cosa en sí. (Kant, 2001) 
Involucrar al estudiante en el contexto de lo observado complejiza la observación. Éste es visto no solamente como un observador de la dinámica que observa; en cambio, su cognición y su estructuración del mapa imponen su sello y recortan el objeto a su perímetro; es decir, considera el observador inmerso dentro del contexto de la acción del conocimiento (Ceberio \& Watzlawick, 1998).

De esta forma el ejercicio de Diseño fija atención particular en el: ¿cómo señalar cuál es la realidad? ¿O existe una realidad verdadera? ¿Qué es lo que se construye, cómo y por qué? Existe un interés constante en hacer lectura de la forma como se construye ese universo personal, partiendo de la estructuración de la realidad, hasta la elaboración de la misma como acto co-constructivo. Un acto "que en estos tiempos de la posmodernidad, aceptar la óptica de la complejidad implica reconocer y respetar la pluralidad de puntos de vista que se traducen en una multiplicidad de modelos interpretativos" (Ceberio \& Watzlawick, 1998, p.16).

Según Alexander (1976), "Cuando hablamos de diseño, el objeto real de la discusión no es solo la forma sino el conjunto que comprende la forma y su contexto”. El proceso de diseño consciente está distante de este conjunto (contexto y necesidad), por lo tanto la forma no es modelada mediante la interacción entre las exigencias del contexto y del usuario real sino mediante una interacción conceptual entre la imagen conceptual del contexto que el diseñador ha aprendido por una parte y diagramas y dibujos que corresponden a las formas, por la otra.

Según Alvis (2007) parece que el diseño se quedó en los postulados y conceptos teóricos que constituyen las profesiones a nivel general; y no se ubica en el desarrollo local de las culturas en las que se encuentra inmerso, pues son los sujetos de esta cultura los que permiten que la materialización conceptual del diseño se lleve a cabo ya que en todas las facetas del proceso de diseño está implícita la comunidad.

Este momento se basa en la interpretación de la información, estimulando el pensamiento sistémico desde una percepción holística de la situación estudiada, comprendiendo elementos y su configuración, identificando problemáticas que puedan transformarse en oportunidades de diseño y finalmente como propuestas de mejoramiento donde los sujetos son activos, dialogan y se relacionan para fortalecer el tejido social. En este sentido es importante partir del reconocimiento de los sujetos involucrados, sus relaciones, sus funciones, sus verdaderas acciones, y el modo cómo estos configuran el sistema.

\section{Los Mapas de Relaciones: red de redes}

Es un modelo que corresponde a la representación esquemática del patrón de organización de la red, manifiesta las relaciones entre los organismos, donde cada nodo en la red representa a cada uno de ellos, y éste a su vez aparece como una red al ser ampliado, y así la trama de la vida está constituida por redes dentro de redes; es decir los nodos de una red se ven como redes más pequeñas. 
Se construye a partir de la base teórico-conceptual que corresponde a las características esenciales del pensamiento sistémico. El primer y más general criterio es el cambio de las partes al todo; los sistemas vivos son totalidades integradas cuyas propiedades no pueden ser reducidas a las de sus partes más pequeñas. Sus propiedades esenciales o «sistémicas» son propiedades del conjunto, que ninguna de las partes tiene por sí sola. Emergen de las «relaciones organizadoras» entre las partes, es decir, de la configuración de relaciones ordenadas que caracteriza aquella clase específica de organismos o sistemas. Otro criterio básico del pensamiento sistémico sería la habilidad para focalizar la atención alternativamente en distintos niveles sistémicos. A través del mundo viviente nos encontramos con sistemas dentro de sistemas. (Capra, 1996, p. 56)
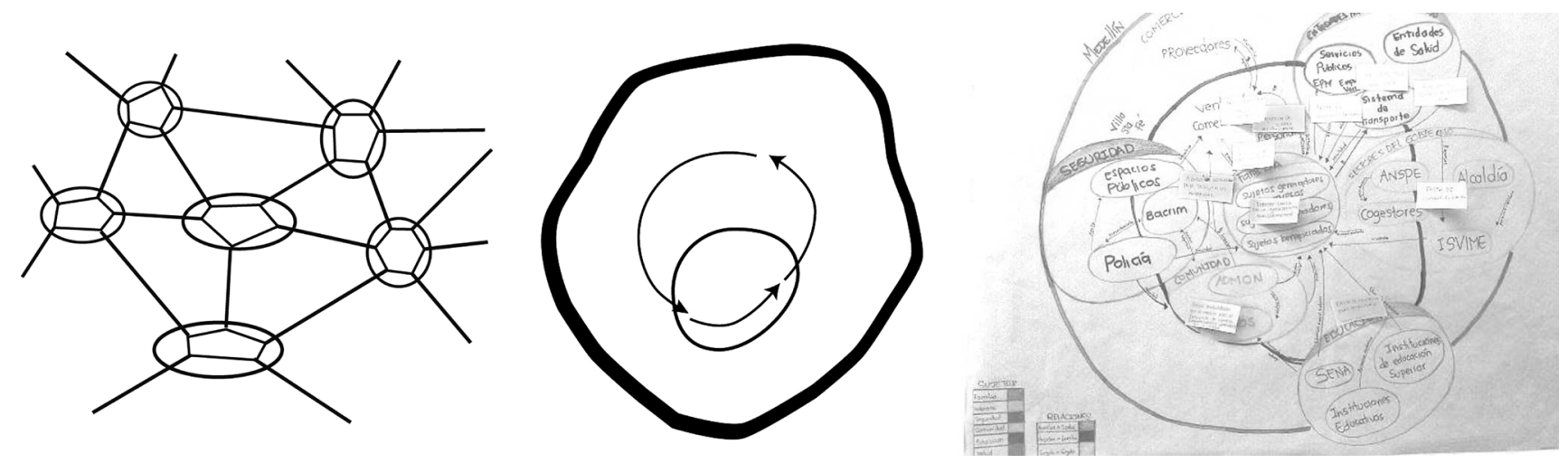

Figura 5. Emulación esquemática del mapa de relaciones

Los mapas de relaciones son como cartografías del sistema alrededor de la situación estudiada, manifiestan un levantamiento de información construida colectivamente del estado actual del sistema, donde el equipo de diseño llega a la definición común del patrón de organización de los sujetos involucrados para tener una visión completa del panorama que sin duda alguna contribuye en la identificación y localización en el sistema actual de situaciones o problemáticas que luego pueden llegar a traducirse en oportunidades de diseño.

Fritjof Capra (1996) afirma que "La percepción del mundo vivo como una red de relaciones (...) ha convertido el pensamiento en términos de red" (p. 58). Esto es una característica esencial del Pensamiento de Diseño en términos de conectividad, relaciones y contexto. Las propiedades esenciales de un sistema de vida surgen de las interacciones y relaciones entre sus partes; es así como en el pensamiento sistémico, la metáfora del conocimiento como construcción, queda reemplazada por la de la red, al percibir la realidad como una red de relaciones. La filosofía de Thich Nhat Hahn considera importante "ofrecer una forma de pensamiento de pedagogía enfatizando en integridad, enfoque holístico, no sólo por el conocimiento en los libros, el conocimiento acerca de vivir en el mundo" (Hooks, 1994, p. 14-15). 
Momento 2: Idear para dar posibles nuevas formas

Este momento está focalizado en hacer al Diseñador Industrial como nuevo sujeto en el sistema que se vincula y propone líneas de mejoramiento en el sistema, donde también asume un rol dentro de él, y es consciente del impacto que generan sus decisiones; éstas de acuerdo a la línea proyectual seleccionada van a generar una nueva representación, con nuevas relaciones, es decir una nueva configuración del sistema, en sí un nuevo sistema.

En esta fase se consolida el pensamiento sistémico como un pensamiento contextual: la explicación en términos de contexto significa la explicación en términos de entorno. Podemos también afirmar que el pensamiento sistémico es un pensamiento medioambiental, una afirmación que en este caso implica visualizar al Diseñador localizado en el entorno como parte de la comunidad.

A partir de la selección de la situación a intervenir se define el Valor, entendido como la característica diferenciadora sobre la que se va a fundamentar la propuesta de Diseño, y la Experiencia, concebida como aquellas emociones a generar en el usuario beneficiado. Estas dos variables articulan en el Concepto del proyecto, que se manifiesta en una frase y luego se transforma en una serie de imágenes que comunican esas definiciones textuales; finalmente alcanza una representación de Maqueta Conceptual donde se depositan y expresan las definiciones ya nombradas. A partir de este momento se desarrollan ejercicios para favorecer la fluidez, originalidad y flexibilidad del pensamiento creativo inspirados en la esencia y significado del concepto como guía en el momento proyectual y llegar de este modo a posibles "nuevas formas".

Momento 3: Configurar para dar formas más definidas

El diseñador se perfila como un facilitador en la comunidad a través del desarrollo de una propuesta objetual inspirada en las características del contexto, y aparecen entonces nuevas configuraciones del sistema. Este momento comprende la definición del diseño manteniendo como directriz la coherencia con el contexto analizado. Se involucra al diseñador en formación a través de la indagación hacia la realidad productiva de la idea de diseño.

Desde la biología y la cultura el ser humano es el resultado de origen y desarrollo evolutivo de las especies con relación al carácter físico del mundo desde un punto individual y colectivo; esto vincula aspectos de la plasticidad, una relación de experiencia que no puede ser suprimida para los seres vivos, ya que corresponde a lo indispensable de contar con el carácter físico de nuestro ambiente. 
De acuerdo con Maldonado (1994) no somos "cerebros metidos en una vasija”. Y aún si lo fuéramos deberíamos de todas formas, en nuestra condición de cerebros, precisamente en nuestra condición de materia pensante, contar con nuestro propio carácter físico y con el carácter físico de la vasija que nos aloja. (p. 15)

\section{Modelo y realidad de proyecto}

La visión sistémica es un elemento transversal que se manifiesta de diferentes formas a través del Proceso de Diseño; como se explica anteriormente el mapa de relaciones es un resultado del primer momento que permite evidenciar el patrón de organización, la configuración de las relaciones entre sus componentes, que determina las características esenciales del sistema.

Este último momento que corresponde a la configuración del Diseño, se consolida el Modelo como la corporeización física de un patrón de organización, lo cual en términos de sistema corresponde a la estructura que implica descripción de los componentes presentes: sus formas, sus composiciones químicas, etc.

Tanto patrón como estructura son dos conceptos que aplican ya sea que el sistema sea vivo o no. Cabe aclarar que el Modelo visto como "sistema no vivo", asigna un carácter fijo a sus componentes ligado a su estructura; mientras que, en un sistema vivo, por el contrario, los componentes cambian continuamente, existe un flujo incesante de materia y energía a través del organismo, cada célula transforma continuamente estructuras y elimina productos de desecho. Tejidos y organismos reemplazan sus células en ciclos continuos, hay crecimiento, desarrollo y evolución.

Para ilustrar la diferencia entre patrón y estructura, tomemos un sistema no vivo bien conocido: una bicicleta. Para que algo pueda ser llamado una bicicleta, deberá existir un número de relaciones funcionales entre sus componentes conocidos como cuadro, pedales, manillar, ruedas, cadena, ruedas dentadas, etc. La configuración completa de estas relaciones funcionales constituye el patrón de organización de la bicicleta. La estructura de la bicicleta es la manifestación física de su patrón de organización en términos de componentes de formas específicas, hechos de materiales específicos. El mismo patrón «bicicleta» puede manifestarse a través de muchas estructuras distintas. El manillar tendrá distinta forma para una bicicleta de paseo, otra de carreras y una de montaña; el cuadro podrá ser pesado y sólido o ligero y delicado, los neumáticos podrán ser estrechos o anchos, tubulares o macizos. Todas estas combinaciones v muchas otras serán reconocidas como diferentes manifestaciones físicas del mismo patrón de relaciones que define a una bicicleta. (Capra, 1996, p. 172) 
La evolución de los sistemas vivos sugiere el proceso como tercer criterio para una completa descripción de la naturaleza de la vida. El proceso vital es la actividad que se ocupa de la continua corporeización del patrón de organización del sistema. Así pues, el criterio de proceso constituye el vínculo entre patrón y estructura. En el caso de nuestra bicicleta, es decir un "sistema no vivo" el patrón de organización está representado por los dibujos de proyecto necesarios para su construcción, la estructura por la bicicleta específica física y el vínculo entre patrón y estructura por el proceso mental de su diseñador.

La concepción del Modelo como un sistema confiere a éste el ser visto como el elemento donde se manifiestan materialmente algunas relaciones, que además permite validar continuamente las interpretaciones de la realidad estudiada y comprender que el proyecto indudablemente también tiene un proceso.

El modelo plástico se llama en algunas lenguas maquette (francés e inglés) y maqueta (español) que son étimos de origen italiano (macchietta). La palabra plástico, evoca, en efecto la idea de una construcción física modelable, en el sentido que le da al escultor cuando trabaja con un material como la arcilla. Esto hace pensar que lo plástico, como sinónimo de maquette, no es algo cerrado, hecho de una vez por todas, sino que se trata, antes bien, de un proceso abierto que se modifica a causa de sucesivas intervenciones, retoques y consideraciones. (Maldonado, 1994, p.150)

El diseñador industrial en su proceso de configuración recurre a modelos plásticos como modo para validar aspectos formales, estructurales o funcionales del Diseño; en esa condición éste cumple propósitos comunicativos además de ser auxiliar en el ejercicio proyectual para responder en términos de forma y estructura a la realidad que quiere interpretar.

En esta exigencia de comunicar el proyecto, de ver anticipadamente, sabiendo que no es el único medio de proyectar. Porque diseñar para proyectar se revela al mismo tiempo como diseñar mientras se proyecta y proyectar mientras se diseña. Es esta co-presencia en interacción entre el medio (diseñar) y el fin (proyectar) lo que permite avanzar hacia la solución buscada y solo a veces hallada. (Maldonado, 1994, p.146)

En el ejercicio que propone el curso, el Diseñador Industrial en formación comprende que el rol del diseñador no se limita simplemente a la propuesta de objetos desde la lectura situaciones o problemáticas, sino que trasciende a una dimensión más compleja; hacia la propuesta de nuevas formas que configuran o re-configuran nuevos sistemas que consolidan la organización de una comunidad. 


\section{Cambios de paradigma}

En síntesis, la teoría completa de los sistemas vivos se fundamenta en el estudio del patrón (forma, orden, cualidad) y el de la estructura (substancia, materia, cantidad); el patrón de organización sólo puede ser reconocido si está corporeizado en una estructura física, siendo éste un proceso continuo en los organismos vivos. "Los tres criterios -patrón, estructura y proceso- son totalmente interdependientes, éstos constituyen tres perspectivas distintas pero inseparables del fenómeno de la vida" (Capra, 1996, p. 173).

El enfoque sistémico de las situaciones, la comprensión de lo complejo de ver el todo con el valor y sentido de sus relaciones requiere una expansión no sólo de nuestras percepciones y modos de pensar, sino también de los valores, contemplados como cambios desde la asertividad a la integración (Capra, 1996). Estas dos tendencias son aspectos fundamentales de todos los sistemas vivos, en términos del deber ser, el aula puede llegar a convertirse en un espacio ideal para propiciar un equilibrio dinámico entre lo asertivo y lo integrativo; pues nuestro entorno en términos de cultura industrial occidental ha evidenciado en lo asertivo sacrificando lo integrativo, lo cual se refleja desequilibradamente en nuestro modo pensamiento y en nuestros valores.

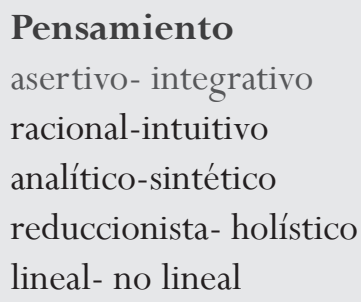

\section{Valores}

asertivo- integrativo

expansión-conservación

competencia-cooperación

cantidad-calidad

dominación-asociación

\footnotetext{
"En la clase no espero que los estudiantes tomen ningún riesgo que yo no tomaría, compartir de una forma que yo no haría"(Hooks, 1994, p. 21).
}

Para quien acompaña el Proceso de Diseño en la figura de profesor se debe acelerar un poco su línea del tiempo, ser más rápido para tener la capacidad de anticiparse a través de la experiencia y de este modo consolidar los procesos dados en el aula. Todo lo que allí pasa debe pasar por cada uno de los individuos, es importante la continua validación de las premisas, construir y de construir las ideas todo el tiempo. Esto implica tomar riesgos e invitar a los estudiantes a hacer lo mismo con sus aprendizajes, porque muy seguramente esto va a ser un asunto importante para aplicar en la vida. 
Referencias

Alexander, C. (1976). Ensayo sobre la síntesis de la forma. Buenos Aires: Ediciones Infinito.

Alvis, J. M. (2007). El diseño industrial como constructo social. Encuentro Latinoamericano de diseño en Palermo (p. 189-191). Buenos Aires: Universidad de Palermo.

Capra, F. (1996). La trama de la vida. Barcelona: Anagrama S.A.

Ceberio, M. R., \& Watzlawick, P. (1998). La construcción del universo. Barcelona: Empresa Editorial Herder.

Kant, I. (2001). Prelegómeno a toda Metafísica futura . Estados Unidos: Hackett Publishing.

Maldonado, T. (1994). Lo real y lo virtual. Barcelona: Gedisa.

Margolin, V. (2002). The Politics of the Artificial. Essays on Design and Design Studies. . The University of Chicago Press.

Figuras

Figura 1. Esquema del patrón de red

Figura 2. Producción de sí mismo, autopoiesis

Figura 3. Causalidad circular en un bucle de retroalimentación

Figura 4. La red en el aula

Figura 5. Emulación esquemática del mapa de relaciones

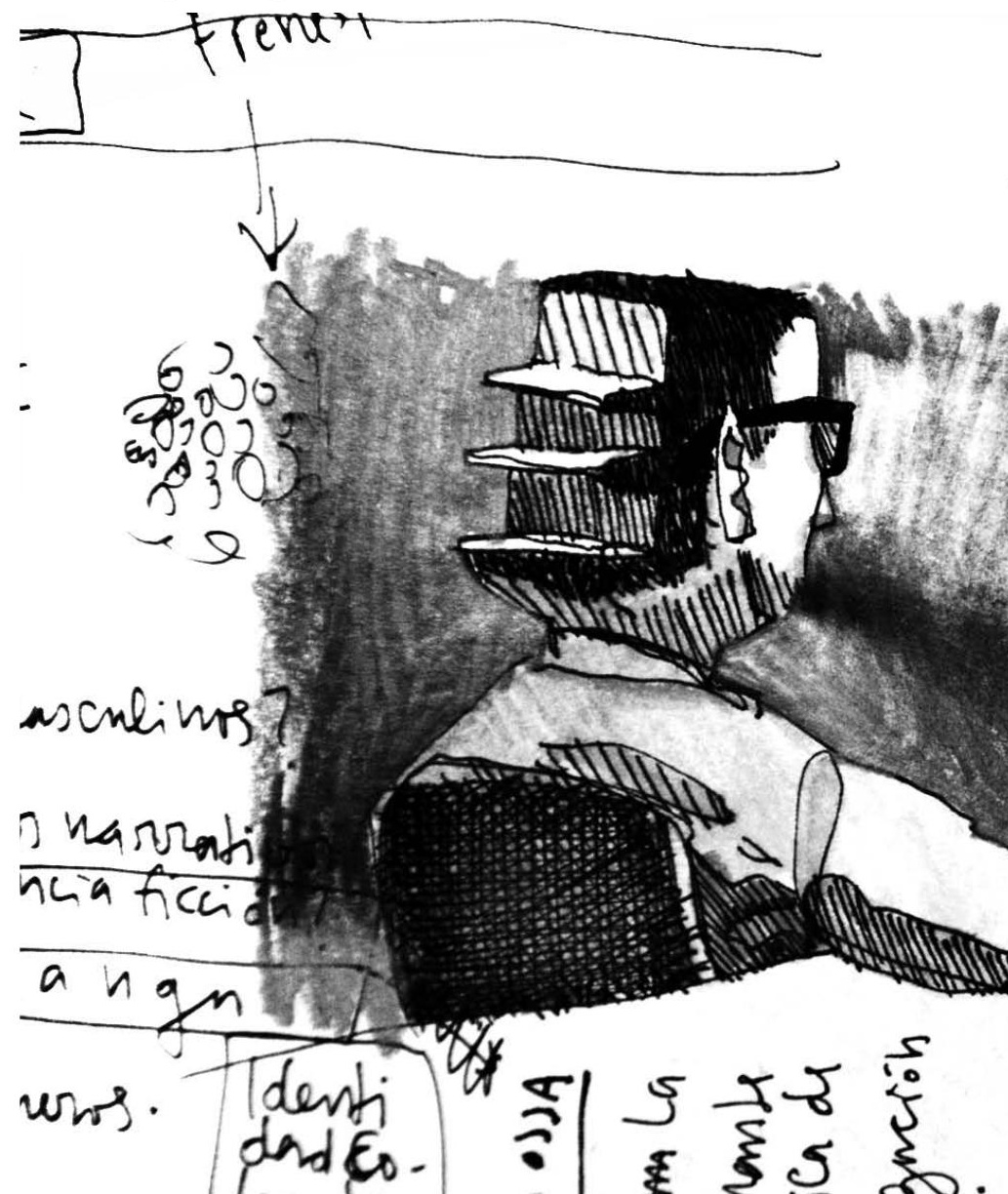

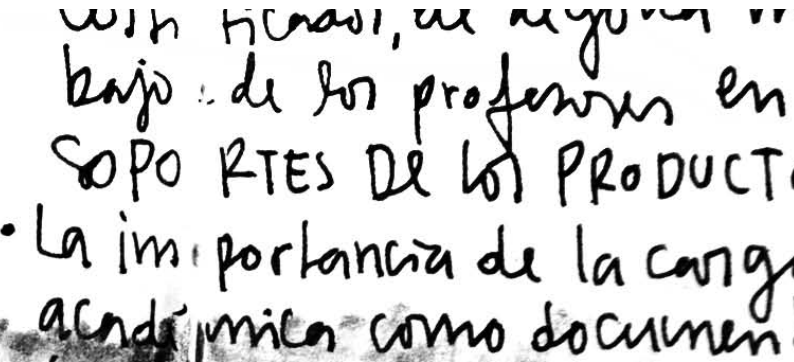
de y mprobación. No abr Proy ectorsen DISEÑo hou visty que wo tienen sopor - Renovación dul CITCE por par

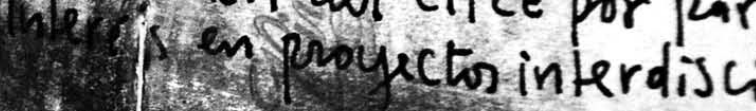

Recibido: ferter 